Research Paper

\title{
Late-course accelerated Hyperfractionation vs. Conventional Fraction Radiotherapy under precise technology plus Concurrent Chemotherapy for Esophageal Squamous Cell Carcinoma: comparison of efficacy and side effects
}

Hongtao Luo ${ }^{1,2}$, Shihong Wei², Xiaohu Wang ${ }^{1,2,5}{ }^{\bowtie}$, Ruifeng Liu², Qiuning Zhang, ${ }^{4,5}$, Zhen Yang3, Zheng $\mathrm{Li}^{4}$, Xiyi $\mathrm{Wei}^{2}$, Yuexiao $\mathrm{Qi}^{2}$, Lijun $\mathrm{Xu}^{2}$

1. The First Clinical Medical College of Lanzhou University, Lanzhou 730000, China.

2. Gansu Provincial Cancer Hospital, Lanzhou 730050, China.

3. The Basic Medical College of Lanzhou University, Lanzhou 730000, China.

4. Lanzhou Heavy Ion Hospital, Lanzhou 730000, China.

5. Institute of Modern Physics, Chinese Academy of Sciences, Lanzhou 730000, China.

$\triangle$ Corresponding author: Prof. Xiaohu Wang, E-mail: xhwanggansu@163.com

(c) The author(s). This is an open access article distributed under the terms of the Creative Commons Attribution License (https://creativecommons.org/licenses/by/4.0/). See http://ivyspring.com/terms for full terms and conditions.

Received: 2019.10.08; Accepted: 2020.02.04; Published: 2020.03.04

\begin{abstract}
Background: The accelerated reproliferation of esophageal squamous cell carcinoma (ESCC) after radiation contributes to conventional fraction radiotherapy (CFRT) failure. Late course accelerated hyperfractionated radiotherapy (LCAHFRT) can improve the long-term survival of esophageal cancer patients in China but is associated with a high rate of side effects due to the large exposure field of two-dimensional treatment and drug toxicity. Intensity-modulated radiotherapy (IMRT) can increase the tumor dose while decreasing the normal tissue dose. Therefore, we compared the outcomes and side effects of LCAHFIMRT plus concurrent chemotherapy (CT) and CFIMRT plus CT for ESCC.

Methods and Materials: Between 2013 and 2016, 114 eligible patients with ESCC were recruited and randomly assigned to receive LCAHFIMRT+CT (58 patients) or CFIMRT+CT (56 patients) by a linear accelerator (6-MV X-ray) under image guidance. Two cycles of CT with cisplatin and docetaxel were also administered.

Results: The complete response (CR) rates were $79.3 \%$ and $61.8 \%$ in the LCAHFIMRT+CT and CFIMRT+CT groups, respectively $(P=0.041)$. The median duration of local control times was $31.0 \pm 1.9$ months for the LCAHFIMRT+CT group and 24.0 \pm 3.3 months for the CFIMRT+CT groups, and the 1-, 2-, and 3 -year local control rates were $86.2 \%, 63.8 \%$, and $41.4 \%$ and $85.7 \%, 51.8 \%$, and $32.1 \%$ for the $\mathrm{LCAHFIMRT}+\mathrm{CT}$ and CFIMRT+CT groups $(\mathrm{P}=0.240)$, respectively. The median survival times were $34.0 \pm 1.1$ months for the LCAHFIMRT+CT group and 28.0.0 \pm 3.7 months for the CFIMRT groups, and the 1-, 2-, and 3-year survival rates were $87.9 \%, 74.1 \%$, and $44.8 \%$ and $87.5 \%, 60.7 \%$, and $39.3 \%$ for the LCAHFIMRT+CT and CFIMRT+CT groups, respectively $(\mathrm{P}=0.405)$. The incidence of side effects was not significantly different between the two groups. Local recurrence and uncontrolled disease resulted in more deaths in the CFIMRT+CT group than in the LCAHFIMRT+CT group (58.9\% vs. 39.7\%) $(P=0.040)$.

Conclusion: For ESCC patients, LCAHFRT delivered by image-guided intensity-modulated techniques Plus Concurrent Chemotherapy with cisplatin and docetaxel keeps safety and high CR rate, as well as local control and long-term survival rates.
\end{abstract}

Key words: Esophageal cancer; late course accelerated hyperfractionation; Intensity-modulated radiotherapy; Image-guided radiotherapy; concurrent chemoradiotherapy 


\section{Introduction}

Esophageal cancer (EC) is the 7th most common malignancy and the $6^{\text {th }}$ most common cause of cancer death around the world; approximately 572,000 new cases of EC and 509,000 deaths related to EC occurred in 2018 [1]. Among these cases, approximately 80\% of the cases of morbidity and mortality occurred in developing countries [2]. In China, the incidence of EC and deaths related to EC account for $50 \%$ of all cases globally around the world and $60 \%$ of those in developing countries. EC is still the main burden of disease for local residents, especially in the rural areas of the Midwest which are considered high-incidence areas $[3,4]$.

Although surgery is an effective treatment for EC, most patients tend to be at an advanced stage at the time of diagnosis [5,6]. Thus, radiotherapy (RT) plays a well-defined role in the management of patients with inoperable locally advanced EC [7]. However, experimental studies have shown that the accelerated re-proliferation of tumor cells after radiation is an important reason for failed conventional fraction radiotherapy (CFRT) [8]. Several clinical trials verified that nonconventional fraction $\mathrm{RT}$, especially late course accelerated hyperfractionated radiotherapy (LCAHFRT), can improve the long-term survival of patients with esophageal cancer [9-11]. In 1999, Shi $\mathrm{XH}$ et al. reported that compared with CFRT, LCAHFRT improved the 5-year survival rate from $26 \%$ to $33 \%$ [10]. Zhao KL et al. used LCAHFRT with concurrent chemotherapy (CT) for patients with ESCC and found that this method was comparable to LCAFRT alone; patients who received concurrent LCAFRT and CT exhibited better survival with a 5 -year survival rate of $40 \%$ and a median survival time of 30.8 months [11]. However, a large number of side effects were reported in these studies due to the large exposure field of two-dimensional treatment and drug toxicity.

Currently, advanced RT techniques such as intensity-modulated radiotherapy (IMRT) and imageguided radiotherapy (IGRT) have progressively changed the practice of treating EC by precisely irradiating the target while minimizing the risk of damaging normal tissues [12-15]. Image-guided intensity-modulated radiotherapy (IG-IMRT) becomes an attractive modality largely owing to the geometrical uncertainties such as respiratory motion and day-to-day position variability of the tumor [16].

The Radiation Therapy Oncology Group (RTOG) phase III intergroup trials RTOG 85-01 and 94-05 showed that RT plus concurrent 5-fluorouracil (5-FU) and cisplatin (DF) is the standard CT regimen for patients with EC $[17,18]$. Novel CT drugs applied in clinical application have significantly improved the curative effects of $\mathrm{CT}$ while alleviating the side effects. Docetaxel is a clinically well-established anti-mitotic CT medication that affects dynamic microtubule assembly and disassembly [19]. Yang HX et al. (2015) reported that the use of neoadjuvant CT agents docetaxel and nedaplatin may provide excellent outcomes for down-staging disease and R0 resection and the patients with EC in their study had a 2-year survival rate of $71.1 \%$ [20].

However, virtually all seminal clinical trials for LCAFRT in EC have utilized two-dimensional radiotherapy (2DRT) or three-dimensional conformal radiotherapy (3DCRT) [9-11,21]. There was a lack of supporting data for LCAFRT in EC under IG-IMRT. Therefore, LCAHFRT delivered by image-guided intensity-modulated techniques in combination with new $\mathrm{CT}$ regimens with cisplatin and docetaxel should be further investigated to assess the efficacy and side effects of this method in ESCC patients.

\section{Materials and Methods}

\section{Eligibility criteria}

The eligibility criteria were as follows: (1) histologically confirmed squamous cell carcinoma of the esophagus; (2) age 18 75 years old with Karnofsky performance status (KPS) scores $\geq 60$; (3) stage I to IV disease according to the 2017 (version 8.0) American Joint Committee on Cancer staging system, with the exception of T4b and M1 disease; (4) life expectancy of at least 6 months; (5) normal baseline laboratory tests (white blood cell count $\geq 3.5 \times 10^{9} / \mathrm{L}$, platelet count $\geq 125 \times 10^{9} / \mathrm{L}$, and hemoglobin $\geq 115$ $\mathrm{g} / \mathrm{L}$ ); (6) normal renal function (serum creatinine $<106$ $\mu \mathrm{mol} / \mathrm{L}$, blood urea $<8.63 \mathrm{mmol} / \mathrm{L}$ ); (7) normal liver function (total serum bilirubin $\leq 20.5 \mu \mathrm{mol} / \mathrm{L}$ and aspartate transaminase and alanine transaminase levels lower than double the upper normal limit); and (8) adequate pulmonary function. The exclusion criteria were as follows: (1) previous treatment with any other therapy, i.e., surgery, CT, or targeted drugs; (2) esophageal perforation or deep ulceration; (3) esophageal bleeding; (4) complete esophageal obstruction; (5) distant metastases; and (6) concomitant serious illness such as uncontrolled angina pectoris, heart failure, interstitial pneumonia, or infection or other diseases contraindicating CT or RT.

\section{Pretreatment evaluations}

The pretreatment evaluation included obtaining a medical history and performing a physical examination, routine blood and biochemistry test, electrocardiogram (ECG) measurements, esophageal barium examination, contrast-enhanced neck and chest computed tomography and ultrasonography of the heart and abdomen, radionuclide bone scan, and 
magnetic resonance imaging (MRI); Positron emission tomography-computed tomography (PET-CT) were used when clinically needed. This study was approved by the Ethics Committee of Gansu Tumor Hospital, Lanzhou, China. Informed consent was provided by the patient or their legal representatives.

\section{Study design}

The patients were randomized into two groups by a random number table. In the study group, the patients received LCAHFIMRT+CT, and in the control group, the patients received CFIMRT+CT.

\section{Radiation and target definition}

Radiation for IMRT was carried out by a linear accelerator (6-MV X-ray). Each patient underwent CT imaging with an intravenous contrast agent for treatment planning. Then, the images were transferred to a radiotherapy planning system (Eclipse). The target area was delineated by two associate chief physicians. The gross tumor volume was defined as the macroscopic primary tumor (GTV) and regional lymph node metastases (GTVnd) based on the endoscopy, endoscopic ultrasonography, barium esophagography and chest CT scans. The clinical target volume (CTV) was defined as the GTV/GTVnd plus a 0.5-1 cm radical margin and 3-4 $\mathrm{cm}$ cranio-caudal margin. The supraclavicular nodes were included as upper esophageal lesions, and the celiac nodes were included as distal esophageal lesions. The planning target volume (PTV) consisted of the CTV plus a $0.5-1 \mathrm{~cm}$ margin for daily setup error and organ motion.

\section{Fractionated dose schemes}

In the present study, all patients received simultaneous integrated boost IMRT (SIB-IMRT) under imaging guided. In the LCAHFIMRT group, the prescribed dose was divided into a two-phase irradiation schedule. The first phase was conventional fractionated irradiation with $44 \mathrm{~Gy} / 2.2 \mathrm{~Gy} / 20$ fractions to the GTV and GTVnd and 36 Gy/1.8 Gy/20 fractions to the CTV with five fractions per week; the second irradiation phase was the accelerated hyperfractionated session. The dose was delivered twice per day with a minimum interval of 6 hours for 10 fractions per week with $18 \mathrm{~Gy} / 1.5 \mathrm{~Gy} / 12$ fractions to the GTV and GTVnd and 13.2 Gy/1.1 Gy/12 fractions to the CTV. The two-phase treatment plan was merged together by the MIM system. The total dose of the two-phase irradiation regimen was 62 Gy for the GTV and GTVnd and 49.2 Gy to the CTV in 32 fractions. In the CFIMRT group, the prescribed dose was 2.2 Gy per fraction for five fractions per week with 61.6 Gy to the GTV and GTVnd and 1.8 Gy per fraction with 50.4 Gy for CTV in 28 fractions for five times a week. For the normal tissue, the percentage of the whole lung volume that received more than 20 Gy irradiation $\left(\mathrm{V}_{20}\right)$ was $\leq 28 \%$, and the percentage that received more than $30 \mathrm{~Gy}\left(\mathrm{~V}_{30}\right)$ was $\leq 20 \%$, the cardiac mean dose (Dmean) was $<30 \mathrm{~Gy}$, and the maximum dose to the medulla spinal was $<45 \mathrm{~Gy}$.

\section{IGRT}

Daily cone beam computed tomography(CBCT) scans (Synergy 4.5, Elekta Ltd, Sweden) were acquired and bony anatomy registration was used for online setup error correction if the error $<1 \mathrm{~cm}$, otherwise, setup was required again when the error $\geq 1 \mathrm{~cm}$.

\section{Chemotherapy}

All patients received two cycles of concurrent CT with a regimen consisting of cisplatin and docetaxel; cisplatin was provided at $25 \mathrm{mg} / \mathrm{m}^{2} /$ day i.v. from days $1-3$, and docetaxel was provided at $60 \mathrm{mg} / \mathrm{m}^{2} /$ day i.v. on day 1 . The first cycle began on the first day of RT, while the second cycle of CT began on the twenty-eighth day of RT if the patients did not exhibit side effects that exceeded grade 2 or if the investigator decided that the patient should not receive the second cycle of CT.

\section{Efficacy evaluation}

The treatment effect was evaluated according to the Response Evaluation Criteria in Solid Tumors (RECIST, version 1.1) after three months of CT and was classified into complete response (CR), partial response $(\mathrm{PR})$, stable disease $(\mathrm{SD})$, and progressive disease (PD) [23]. Long-term effects were evaluated by the median local control time, median survival time, 1-, 2- and 3-year local control rates and 1-, 2- and 3 -year survival rates.

\section{Side effect criteria}

The acute and long-term side effects were evaluated by the American Radiation Therapy Oncology Group (RTOG) criteria severity scales (http:// www.rtog.org./). Acute side effects appeared between the beginnings of treatment to three months after completing treatment. Long-term side effects were recorded at each patient follow-up visit.

\section{Statistical analysis}

The data were analyzed using SPSS 21.0 software. The constituent ratio was analyzed by the $x^{2}$ test, and the measurement data were analyzed by the nonparametric test; the total survival rate and local control rate were calculated by the Kaplan-Meier method, and the significance of the differences was examined by the log-rank method. A P value less than 0.05 indicated statistical significance. 


\section{Results}

\section{Patient characteristics}

Between January 2013 and June 2016, 114 patients with esophageal squamous cell carcinoma (ESCC) were randomized to the study. The pretreatment characteristics of the 114 eligible and assessable patients are listed in Table 1 . The two randomized groups were well balanced regarding sex, age, tumor location, primary tumor length, tumor stage, nodal stage, clinical stage, histological grade, KPS score and weight loss before treatment.

Table 1. Clinical characteristics of esophageal cancer patients $n$ (\%)

\begin{tabular}{|c|c|c|c|c|}
\hline Feature & $\begin{array}{l}\text { LCAHFIMRT+CT } \\
\text { group } \\
58 \text { case }\end{array}$ & $\begin{array}{l}\text { CFIMRT+CT } \\
\text { group } \\
56 \text { case }\end{array}$ & $x^{2}$ & $\mathrm{P}$ \\
\hline \multicolumn{3}{|l|}{ Gender } & 0.386 & 0.535 \\
\hline Male & $34(58.6 \%)$ & $36(64.3 \%)$ & & \\
\hline Female & $24(41.4 \%)$ & $20(35.7 \%)$ & & \\
\hline \multicolumn{3}{|l|}{ Age (years) } & 1.723 & 0.423 \\
\hline$\leq 50$ & $15(25.9 \%)$ & $12(21.4 \%)$ & & \\
\hline $50 \sim 70$ & $24(41.4 \%)$ & $30(53.6 \%)$ & & \\
\hline$\geq 70$ & $19(32.8 \%)$ & $14(25.0 \%)$ & & \\
\hline \multicolumn{3}{|c|}{ Tumor location } & 1.459 & 0.482 \\
\hline Upper & $22(37.9 \%)$ & $19(33.9 \%)$ & & \\
\hline Middle & $17(29.3 \%)$ & $22(39.3 \%)$ & & \\
\hline lower & $19(32.8 \%)$ & $15(26.8 \%)$ & & \\
\hline \multicolumn{3}{|c|}{ Primary tumor length } & 0.860 & 0.354 \\
\hline$<5 \mathrm{~cm}$ & $17(29.3 \%)$ & $21(37.5 \%)$ & & \\
\hline$\geq 5 \mathrm{~cm} \sim<10$ & $41(70.7 \%)$ & $35(62.5 \%)$ & & \\
\hline \multicolumn{3}{|l|}{ Tumor stage } & 2.277 & 0.517 \\
\hline $\mathrm{T} 1$ & $5(8.6 \%)$ & $4(7.1 \%)$ & & \\
\hline $\mathrm{T} 2$ & $22(37.9 \%)$ & $28(50.0 \%)$ & & \\
\hline T3 & $29(50.0 \%)$ & $21(37.5 \%)$ & & \\
\hline T4a & $2(3.4 \%)$ & $3(5.4 \%)$ & & \\
\hline \multicolumn{3}{|l|}{ Nodal stage } & 1.245 & 0.537 \\
\hline No & $38(65.5 \%)$ & $37(66.1 \%)$ & & \\
\hline N1 & $6(10.3 \%)$ & $9(16.1 \%)$ & & \\
\hline N2 & $14(24.1 \%)$ & $10(17.9 \%)$ & & \\
\hline \multicolumn{3}{|c|}{ Clinical stage } & 0.307 & 0.998 \\
\hline I & $4(6.9 \%)$ & $4(7.1 \%)$ & & \\
\hline IIB & $17(29.3 \%)$ & $16(28.6 \%)$ & & \\
\hline IIA & $16(27.6 \%)$ & $16(28.6 \%)$ & & \\
\hline IIIA & $9(15.5 \%)$ & $8(14.3 \%)$ & & \\
\hline IIIB & $10(17.2 \%)$ & $9(16.1 \%)$ & & \\
\hline IVA & $2(3.4 \%)$ & $3(5.4 \%)$ & & \\
\hline \multicolumn{3}{|c|}{ Histological grade } & 3.181 & 0.204 \\
\hline well & $22(37.9 \%)$ & $19(33.9 \%)$ & & \\
\hline moderately & $32(55.2 \%)$ & $27(48.2 \%)$ & & \\
\hline poorly & $4(6.9 \%)$ & $10(17.9 \%)$ & & \\
\hline \multicolumn{3}{|l|}{ KPS } & 1.417 & 0.234 \\
\hline$\geq 60 \sim \leq 770$ & $26(44.8 \%)$ & $19(33.9 \%)$ & & \\
\hline$\geq 80$ & $32(55.2 \%)$ & $37(66.1 \%)$ & & \\
\hline \multicolumn{3}{|c|}{ Weight loss before therapy } & 1.698 & 0.193 \\
\hline$<10 \%$ & $39(67.2 \%)$ & $31(55.4 \%)$ & & \\
\hline$\geq 10 \%$ & $19(32.8 \%)$ & $25(44.6 \%)$ & & \\
\hline
\end{tabular}

*LCAHFIMRT: late course accelerated hyperfractionated intensity modulated radiotherapy; CFIMRT: conventional fraction intensity modulated radiotherapy; CT: chemotherapy.

\section{Treatment and follow-up}

In the LCAHFIMRT+CT group, the highest radiation dose to the GTV or GTVnd was $62 \mathrm{~Gy}$, and the minimum dose was $52 \mathrm{~Gy}$; the corresponding values were $61.6 \mathrm{~Gy}$ and $55 \mathrm{~Gy}$ in the CFIMRT+CT group. All patients in both groups completed two cycles of CT. The patients were followed up every three months for two years, then every six months for two years, and then every year. The last follow-up occurred in June 2019. The follow-up periods were 36-58 (median 45) months, and the follow-up rate was $100 \%$.

\section{Short-term curative effect}

The CR, PR, SD and PD were 79.3\%, 10.3\%, 6.9\% and $3.4 \%$ in the LCAHFIMRT+CT group and $61.8 \%$, $23.2 \%, 10.7 \%$ and $5.4 \%$ in the CFIMRT+CT group, respectively; the $\mathrm{CR}$ was significantly different between the LCAHFIMRT+CT group and CFIMRT+ CT group ( $\mathrm{P}=0.041)$, but the $\mathrm{PR}, \mathrm{SD}$ and $\mathrm{PD}$ were not significantly different between the two groups ( $P=0.065,0.417$ and 0.619 , respectively), as shown in Table 2 .

Table 2. Comparison of short-term curative effects $n$ (\%)

\begin{tabular}{|c|c|c|c|c|}
\hline Feature & $\begin{array}{l}\text { LCAHFIMRT+CTgroup } \\
58 \text { case }\end{array}$ & $\begin{array}{l}\text { CFIMRT+CTgroup } \\
56 \text { case }\end{array}$ & $x^{2}$ & $\mathrm{P}$ \\
\hline CR & $46(79.3 \%)$ & $34(61.8 \%)$ & 4.178 & 0.041 \\
\hline PR & $6(10.3 \%)$ & $13(23.2 \%)$ & 3.398 & 0.065 \\
\hline SD & $4(6.9 \%)$ & $6(10.7 \%)$ & 0.519 & 0.471 \\
\hline PD & $2(3.4 \%)$ & $3(5.4 \%)$ & 0.248 & 0.619 \\
\hline
\end{tabular}

\section{Local control rate and survival rate}

The median local control times were 31.0 1.9 months (95\% CI 27.3-34.7) in the LCAHFIMRT+CT group and $24.0 \pm 3.3$ months (95\% CI 17.5-30.5) in the CFIMRT+CT group, respectively; the 1-, 2-, and 3-year local control rates were $86.2 \%, 63.8 \%$, and $41.4 \%$ in the LCAHFIMRT+CT group and $85.7 \%, 51.8 \%$, and $32.1 \%$ in the CFIMRT+CT group, respectively. The differences between the two groups were not significant (Fig. $1, X^{2}=1.383, \mathrm{P}=0.240$ ). The median survival times were $34.0 \pm 1.1$ months $(95 \%$ CI 31.9-36.1) in the LCAHFIMRT+CT group and 28.0 \pm 3.7 months (95\% CI 20.7-35.3) in the CFIMRT group, respectively. The 1-, 2-, and 3-year survival rates were $87.9 \%, 74.1 \%$, and $44.8 \%$ in the LCAHFIMRT+CT group and $87.5 \%, 60.7 \%$, and $39.3 \%$ in the CFIMRT+ CT groups, respectively. The differences between the two groups were not significant (Fig. 2; $X^{2}=0.693$, $\mathrm{P}=0.405)$.

\section{Side effects of treatment}

The incidence of acute and late side effects in the LCAHFIMRT+CT group was slightly higher than the CFIMRT+CT group. Because nutritional supplementation and symptomatic treatment were administered 
to patients throughout the study period, the main side effects were below grade 3 . In terms of severe side effects: 2 cases of esophageal perforation and 2 cases of pulmonary fibrosis were noted in the LCAHFIMRT+CT group, whilst 1 case of esophageal perforation and 3 cases of pulmonary fibrosis were noted in the CFIMRT+CT group, which was not found to be a significant difference $(P>0.05)$ (Table 3$)$.
Table 3. Comparison of treatment side effects

\begin{tabular}{lllll}
\hline Reasons of death & \multicolumn{2}{l}{ Group } & \multirow{2}{*}{$X^{2}$} & $\mathrm{P}$ \\
\cline { 2 - 3 } & LCAHFIMRT+CT & CFIMRT+CT & & \\
\hline Number of cases & 43 & 45 & & \\
Local recurrence or & $23(39.7 \%)$ & $33(58.9 \%)$ & 4.235 & 0.040 \\
Uncontrolled disease & & & & \\
Distant metastasis & $15(25.9 \%)$ & $22(39.3 \%)$ & 2.342 & 0.126 \\
Therapeutic side effects & $8(13.8 \%)$ & $7(12.5 \%)$ & 0.042 & 0.838 \\
\hline
\end{tabular}

*LCAHFIMRT: Late course accelerated hyperfractionated intensity modulated radiotherapy; CFIMRT: conventional fraction intensity modulated radiotherapy;

CT: chemotherapy.

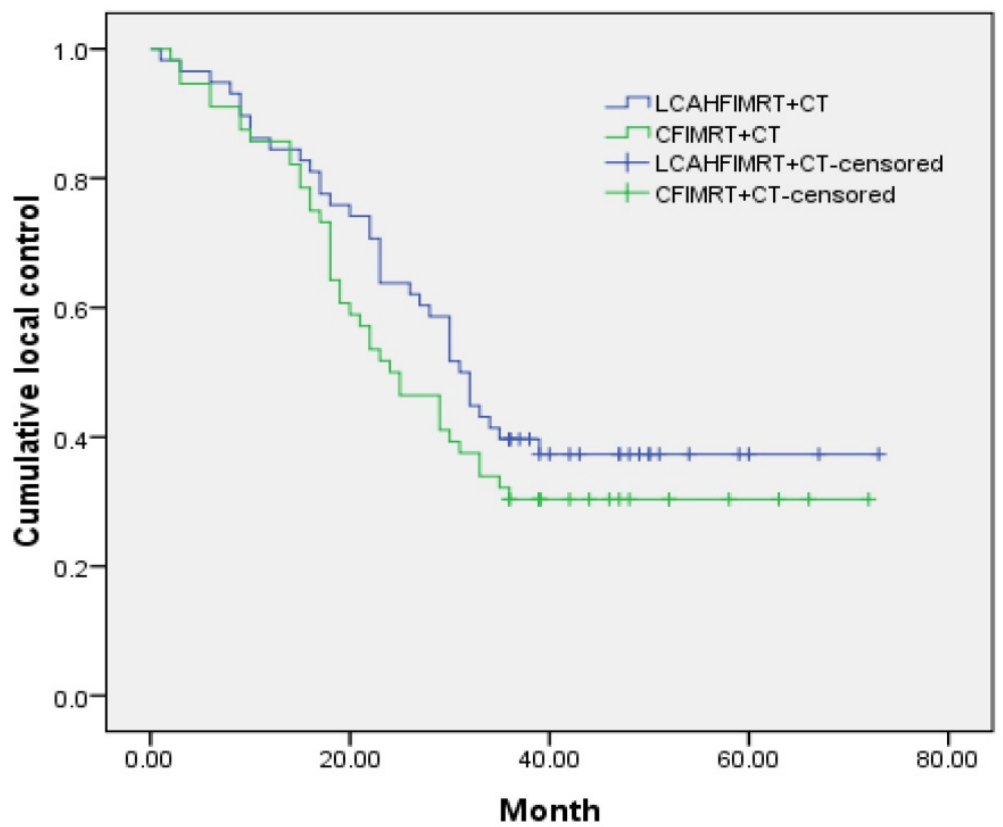

Figure 1. Comparison of local control rates. The 1-, 2-, and 3-year-local control rate curves (data shown in months) are compared in the LCAHFIMRT+CT group and the CFIMRT $+C T$ group of ESCC.

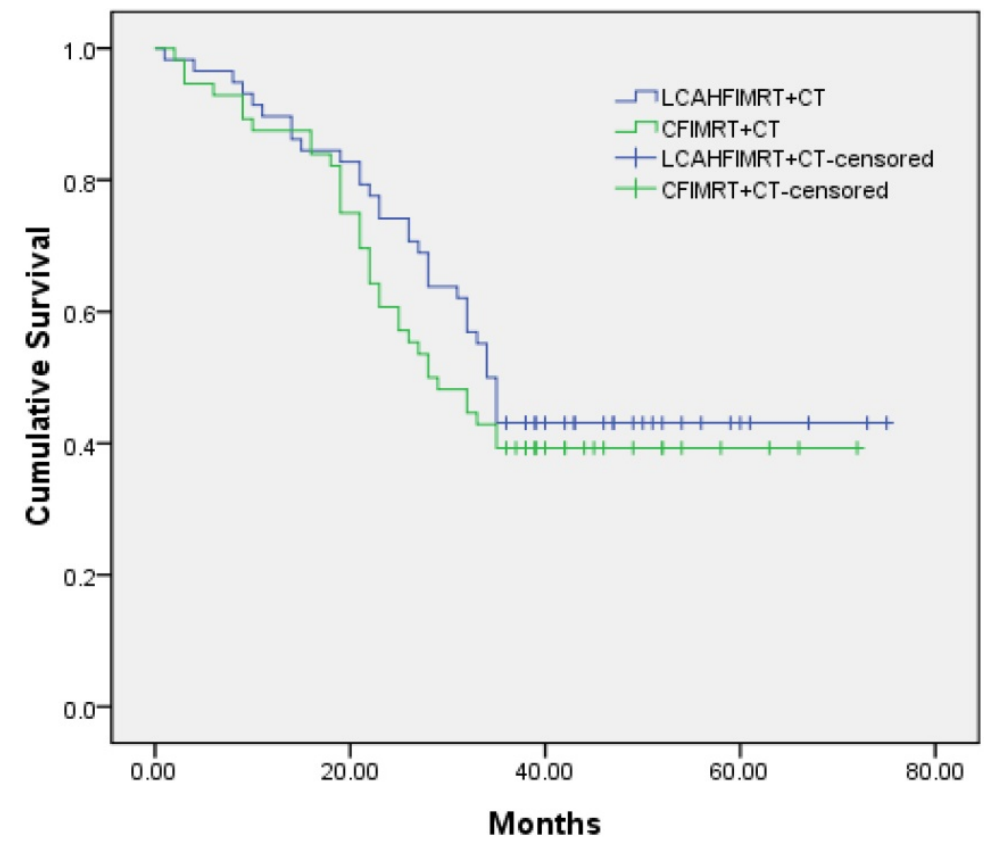

Figure 2. Comparison of survival rates. The 1-, 2-, and 3-year survival rate curves (data shown in months) are compared in the LCAHFIMRT+CT group and the CFIMRT+CT group of ESCC. 


\section{Cause of death}

At the last follow-up, A total of 43 patients died in the LCAHFIMRT+CT group, due to the following: local recurrence or uncontrolled disease $39.7 \%$ (23/ 58); distant metastasis $25.9 \%$ (15/58); and therapeutic side effects $13.8 \%(8 / 58)$. A total of 45 patients died in the CFIMRT+CT group due to the following: local recurrence or uncontrolled disease $58.9 \%$ (33/56); distant metastasis $39.3 \%(22 / 56)$; and therapeutic side effects $12.5 \%(7 / 56)$. The incidence of local recurrence or uncontrolled disease was significantly different between the two groups $(\mathrm{P}=0.040)$, but the incidences of distant metastasis and therapeutic side effects were not $(\mathrm{P}=0.126$ and 0.838 , respectively). The data are shown in Table 4.

Table 4. Comparison of the causes of death, $n(\%)$

\begin{tabular}{|c|c|c|c|c|}
\hline \multirow[t]{2}{*}{ Reasons of death } & \multicolumn{2}{|l|}{ Group } & \multirow[t]{2}{*}{$x^{2}$} & \multirow[t]{2}{*}{$\mathrm{P}$} \\
\hline & LCAHFIMRT+CT & CFIMRT+CT & & \\
\hline Number of cases & 43 & 45 & & \\
\hline $\begin{array}{l}\text { local recurrence or } \\
\text { uncontrolled disease }\end{array}$ & $23(39.7 \%)$ & $33(58.9 \%)$ & 4.235 & 0.040 \\
\hline Distant metastasis & $15(25.9 \%)$ & $22(39.3 \%)$ & 2.342 & 0.126 \\
\hline Therapeutic side effects & $8(13.8 \%)$ & $7(12.5 \%)$ & 0.042 & 0.838 \\
\hline
\end{tabular}

*LCAHFIMRT: Late course accelerated hyperfractionated intensity modulated radiotherapy; CFIMRT: conventional fraction intensity modulated radiotherapy; CT: chemotherapy.

\section{Discussion}

Radiation plays a crucial role in the treatment of EC; however, the 5-year survival rate of EC patients treated with RT alone is $23.31 \pm 10.21 \%$ due to the high rates of failed local recurrence and uncontrolled disease [7]. The accelerated reproliferation of tumor cells after RT is another important reason for RT failure [8]. In a 2DRT era, Zhao KL et al. reported that LCAHFRT together with CT using the DF regimen led to a median survival of 30.8 months and 1-, 3-, and 5 -year survival rates of $67 \%, 44 \%$, and $40 \%$, respectively [11]. Wang JH et al. showed that the 1-, $2-$, and 3-year local control rates and survival rates of 3-dimensional late course accelerated hyperfractionated conformal radiotherapy were $81.3 \%, 62.5 \%$, and $50 \%$ and $79.2 \%, 56.3 \%$, and $43.8 \%$, respectively[21]. Thus, high-dose radiation did not lead to improved curative effects for EC patients. In the present study, all patients received image-guided conformal IMRT, which utilizes computer-controlled linear accelerators to deliver precise radiation doses to the GTV and GTVnd as well as CT-based image guidance and repositioning prior to $\mathrm{RT}$. Li CC et al. [23] showed that concurrent chemoradiation (CCRT) coupled with IGRT is associated with better overall survival compared with CCRT without IGRT in patients with nonoperable localized ESCC.
The phase III RTOG intergroup trials 85-01 and 94-05 established the value of CCRT as the standard therapy for patients with EC patients $[17,18]$, and the DF regimen was recommended as the first-line CT for EC [24]. However, the DF regimen has limited efficacy and application due to the mucosal response to 5-FU. Compared to the DF regimen, docetaxel combined with cisplatin had synergistic effects at lower concentrations and promoted apoptosis but did not increase the side effects of CT [25]. Sasaki K et al. used neoadjuvant chemoradiotherapy with docetaxel/ cisplatin/5-FU (DCF) in 30 patients with ESCC. The 3 -year overall survival rate was $62.2 \%$, and the 3 -year pathologic complete response $(\mathrm{pCR})$ rate was $84 \%$ [26]. Combination chemotherapy using DCF has shown promising efficacy for patients with ESCC in adjuvant and salvage settings [27, 28]. In our study, we used LCAHFIMRT plus CT in view of the high dose of radiotherapy for patients and a two-drug regimen with docetaxel and cisplatin (DC) for concurrent chemotherapy. The results showed that the $\mathrm{CR}$ rate was $79.3 \%$ in the LCAHFIMRT+CT group, which was higher than that in the CFIMRT $+\mathrm{CT}$ group (61.8\%). Compared to CFIMRT+CT, LCAHFIMRT+ CT prolonged the median local control time and the median survival time of patients. The 1-, 2-, and 3 -year local control rates and survival rates were $86.2 \%, 63.8 \%$, and $41.4 \%$ and $87.9 \%, 74.1 \%$, and $44.8 \%$, respectively, in the LCAHFIMRT+CT group, which were higher than those in the CFIMRT+CT group as well as those reported in previous studies. The acute and late side effects that occurred were mainly grade I and II in both the LCAHFIMRT+CT group and the CFIMRT+CT group, possibly because IMRT reduces the radiation dose to the surrounding normal tissue and because not all patients received adjuvant CT after CCRT in our study. However, we observed 6 cases and 5 cases of fatal serious side effects in the esophagus and lung in the LCAHFIMRT+CT group and the CFIMRT+CT group, respectively. The main causes of death were local recurrence or uncontrolled disease in both groups.

Overall, our results revealed that image-guided LCAHFIMRT combined with new CT regimens with cisplatin and docetaxel, which had a better short-term efficacy and a slightly higher local control rates and long-term survival rates than CFIMRT for ESCC. The side effects in the LCAHFIMRT+CT group were similar to that in the CFIMRT group owing to use the IG-IMRT and new chemotherapy regimens. However, improving the local control rate and reducing the long-term side effects of CCRT are still difficult problems to solve in clinical practice. 


\section{Acknowledgments}

This work was supported by grants from the 2017 Lanzhou Talent Innovation and Entrepreneurship Project Funding, Key Technologies for the Basic and Clinical Application of Domestic Heavy Ion Accelerator for Tumor Treatment (grant number 2017RC-23), The Young Creative Talents of Gansu Project in Gansu Province (grant number [2016] 97), The Science and Technology Support Program of Gansu Provincial Science and Technology Department (grant number [2016] 1604FKCA109) and the Gansu Provincial Health sector management research project (grant number [2019] 31).

\section{Author Contributions}

(I) Conception and design: Hongtao Luo, Xiaohu Wang; (II) Administrative support: Xiaohu Wang, Hongtao Luo, Shihong Wei; (III) Experiment operation: Hongtao Luo, Shihong Wei, Ruifeng Liu, Xiyi Wei, Yuexiao Qi ,Lijun Xu; (IV) Data analysis and interpretation: Hongtao Luo, Qiuning Zhang, Zhen Yang, Zheng Li, Ruifeng Liu.

\section{Competing Interests}

The authors have declared that no competing interest exists.

\section{References}

1. Bray F, Ferlay J, Soerjomataram I, Siegel RL, Torre LA, Jemal A. Global cancer statistics 2018: GLOBOCAN estimates of incidence and mortality worldwide for 36 cancers in 185 countries. CA: a cancer journal for clinicians. 2018; 68:394-24.

2. Torre LA, Bray F, Siegel RL, Ferlay J, Lortet-Tieulent J, Jemal A. Global cancer statistics, 2012. CA: a cancer journal for clinicians. 2015; 65:87-08.

3. Chen W, Zheng R, Baade PD, Zhang S, Zeng H, Bray F, et al. Cancer statistics in China, 2015. CA: a cancer journal for clinicians. 2016;66:115-32.

4. Lin Y, Totsuka Y, Shan B, Wang C, Wei W, Oiao Y, et al. Esophageal cancer in high-risk areas of China: research progress and challenges. Annals of epidemiology. 2017; 27:215-21.

5. Fransen LFC, Luyer MDP. Effects of improving outcomes after esophagectomy on the short- and long-term: a review of literature. Journal of thoracic disease. 2019; 11:S845-50.

6. Morita M, Yoshida R, Ikeda K, Egashira A, Oki E, Sadanaga N, et al. Advances in esophageal cancer surgery in Japan: an analysis of 1000 consecutive patients treated at a single institute. Surgery. 2008;143:499-08.

7. Zhou ZG, Gao XS, Qiao XY, Zhang P. Literature analysis of radiotherapy for esophageal cancer in China. Chinese Journal of Cancer.2010;29:873-81.

8. Withers HR, Taylor JMG, Maciejewski B. The hazard of accelerated tumor clonogen repopulation during radiotherapy. Acta Oncol. 1988;27:131-46.

9. Jeremic B, Shibamoto Y, Acimovic L, Matovic Z, Milicic B, Milisavljevic S, et al. Accelerated hyperfractionated radiation therapy and concurrent 5-fluorouracil/cisplatin chemotherapy for locoregional squamous cell carcinoma of the thoracic esophagus: a phase II study. Int J Radiat Oncol Biol Phys. 1998; 40:1061-66.

10. Shi $\mathrm{XH}, \mathrm{Yao} \mathrm{W}$, Liu T. Late course accelerated fractionation in radiotherapy of esophageal carcinoma. Radiother Oncol. 1999; 51:21-26.

11. Zhao KL, Shi XH, Jiang GL, Yao WQ, Guo XM, Wu GD, et al. Late course accelerated hyperfractionated radiotherapy plus concurrent chemotherapy for squamous cell carcinoma of the esophagus: a phase III randomized study. Int J Radiation Oncology Biol Phys. 2005; 62:1014-20.

12. Roeder F, Nicolay NH, Nouyen T, Saleh-Ebrahimi L, Askoxylakis V, Bostel T, et al. Intensity modulated radiotherapy (IMRT) with concurrent chemotherapy as definitive treatment of locally advanced esophageal cancer. Radiation Oncology. 2014:1-9.

13. Chen J, Su T, Lin Y, Wang B, Li J, Pan J, Chen C. Intensity-modulated radiotherapy combined with paclitaxel and platinum treatment regimens in locally advanced esophageal squamous cell carcinoma. Clin Transl Oncol. 2018; 20:411-19.
14. Vosmik M, Petera J, Sirak I, Hodek M, Paluska P, Dolezal J, et al. Technological advances in radiotherapy for esophageal cancer. World J Gastroenterol. 2010; 16:5555-64.

15. Nabavizadeh N, Elliott DA, Chen Y, Kusano AS, Mitin T, Thomas CR, et al Image Guided Radiation Therapy (IGRT) Practice Patterns and IGRT's Impact on Workflow and Treatment Planning: Results From a National Survey of American Society for Radiation Oncology Members. Int J Radiat Oncol Biol Phys 2016; 94:850-57.

16. Voncken FEM, Nakhaee S, Stam B, Wiersema L, Vollenbrock SE, Dieren JMV, et al. Quantification of esophageal tumor motion and investigation of different image-guided correction strategies. Journal Pre-proof. 2019; S1879-8500(19): 30361-63

17. Cooper JS, Guo MD, Herskovic A, Macdonald JS, Martenson JA, Al-Sarraf M, et al. Chemoradiotherapy of locally advanced esophageal cancer: long-term follow-up of a prospective randomized trial (RTOG 85-01). Radiation Therapy Oncology Group. JAMA 1999; 281:1623-27.

18. Minsky BD, Pajak TF, Ginsberg RJ, Pisansky TM, Martenson J, Komaki R, et al. INT 0123 (Radiation Therapy Oncology Group 94-05) phase III trial of combined-modality therapy for esophageal cancer: high-dose versus standard-dose radiation therapy. J Clin Oncol. 2002; 20:1167-74.

19. Lyseng-Williamson KA, Fenton C. Docetaxel: a review of its use in metastatic breast cancer. Drugs. 2005; 65:2513-31.

20. Yang HX, Yao J, Wen H, Yu L, Liu W, Liang H, et al. Clinical Evaluations of Neoadjuvant Chemotherapy With DN and FP Regimens for Patients With Middle or Lower Thoracic Locally Advanced Esophageal Squamous Cell Carcinoma. Zhonghua Yi Xue Za Zhi. 2015; 95 (19), 1530-3.

21. Wang JH, Lu XJ, Zhou J, Wang F. A Randomized Controlled Trial of Conventional Fraction and Late Course Accelerated Hyperfraction Three-Dimensional Conformal Radiotherapy for Esophageal Cancer. Cell Biochem Biophys. 2012; 62:107-12.

22. Eisenhauer EA, Therasse P, Bogaerts I Schwartz LH, Sargent D, Ford R, et al. New response evaluation criteria in solid tumours: revised RECIST guideline (version 1.1). European journal of cancer (Oxford, England: 1990). 2009; 45:228-47.

23. Li CC, Chen CY, Chien CR. Comparative effectiveness of image-guided radiotherapy for non-operated localized esophageal squamous cell carcinoma patients receiving concurrent chemoradiotherapy: A populationbased propensity score matched analysis. Oncotarget. 2016; 7:71548-55.

24. Shibata A, Matsuda T, Ajiki W, Sobue T. Trendin incidence of adeno-carcinoma of the esophagus in Japan,1993-2001. Jpn J Clin Oncol. 2008; 38:464-68.

25. Tao H, Tang X, Jin L, Zhao Y, Luo Y, Zhang Z, et al. Synergistic effect of docetaxel combined with cisplatin on inhibiting human osteosarcoma in nude mice. Biochem Biophys Res Commun. 2018; 505:372-77.

26. Sasaki $K$, Uchikado $Y$, Omoto $I$, Arigami $T$, Osako $Y$, Noda $M$, et al. Neoadjuvant chemoradiotherapy with docetaxel, cisplatin, and 5-fluorouracil (DCF-RT) for locally advanced esophageal squamous cell carcinoma. Cancer Chemother Pharmacol. 2019; 83:581-87.

27. Sugawara M, Katada N, Takahashi K, Higuchi K, Komori S, Moriya H, et al. Retrospective evaluation of adverse events of neoadjuvant or induction chemotherapy with docetaxel, cisplatin, and 5-fluorouracil in esophageal squamous cell carcinoma. Esophagus. 2013, 10:65-69.

28. Tamura S, Imano M, Takiuchi H, Kobayashi $K$, Furukawa $H$, Miki H, et al. Phase II study of docetaxel, cisplatin and 5-fluorouracil (DCF) for metastatic esophageal cancer (OGSG 0403). Anticancer Res. 2012; 32:1403-08. 